\title{
The Impact of Frequency-Agility on Dynamic Spectrum Sharing
}

\author{
Lili Cao, Lei Yang and Haitao Zheng \\ Department of Computer Science \\ University of California, Santa Barbara \\ \{lilicao, leiyang, htzheng\}@cs.ucsb.edu
}

\begin{abstract}
Designed to adapt spectrum usage on-the-fly, frequency-agile radios can drastically improve performance of wireless networks. Such flexibility, however, comes with a cost of increased hardware complexity. This motivates us to understand when and why having higher degree of frequency-agility helps and how much improvement it can lead to. In this paper, we approach this question by comparing two types of agile radios in the context of dynamic spectrum sharing in any given spectrum chunk. We consider 1-agile radios that use a single frequency channel but can adjust the channel's width and central frequency, and $k$-agile radios that can combine up to $k$ non-contiguously aligned frequency segments into one transmission. We show that, due to inherent demand dynamics and conflict heterogeneity, networks using 1-agile radios often face the problem of spectrum fragmentation. But $k$-agile radios can effectively suppress this problem directly at the physical layer. Using theoretical analysis and simulation experiments, we quantify the advantage of $k$ agile radios over 1-agile radios in their network spectrum usage. For a fair comparison, we abstract the impact of demand and topology configurations by evaluating the worst case and average case performance. Our results show that in worst cases, the improvement of using fully-agile radios is arbitrarily large, although the improvement of using $k$-agile radios is upper bounded by $k$. In average cases, the improvement reduces to 10-40\% under typical network configurations. Interestingly, in the context of dynamic spectrum sharing, 2-agile radios realize the majority of the improvement brought by fully-agile radios.
\end{abstract}

\section{INTRODUCTION}

The introduction of software defined radios and cognitive radios has led to a fast growing interest in "frequency-agile" radios. An agile radio can adapt on-the-fly the frequency location and the amount of its spectrum usage. Such flexibility opens up new exciting functionalities for wireless devices, particularly in the area of dynamic spectrum access [1]. For example, infrastructure access points can vary the amount of spectrum usage proportional to their present traffic volumes, achieving network-wide load balancing and improving user satisfaction [2], [3].

In this paper, we consider a specific form of frequencyagility and its impact on dynamic spectrum access. For a given spectrum band, the frequency-agility is defined as the flexibility in using the spectrum band. Fig. 1 presents two types of frequency-agile radios in terms of their spectrum usage pattern. Both radios partition the spectrum band finely into many sections (e.g. OFDMA subcarriers). The first type, 1agile radios, can build a transmission using any single chunk of frequency in this band. Using OFDMA, each chunk is a (a) 1-agile radios

(b) k-agile radios

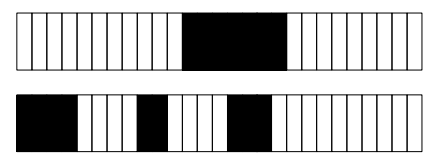

Fig. 1. Comparing the two types of frequency-agile radios. Both radios partition the available spectrum into a large number of segments. 1-agile radios can use only one chunk while $k$-agile radios can use up to $k$ chunks.

collection of contiguously aligned subcarriers. A prototype of 1 -agile radios is available now by modifying commodity WiFi radios to change channel width between $5,10,20$, and $40 \mathrm{MHz}$ [4]. The second and more advanced type is $k$-agile radios, where each radio can use up to $k$ frequency chunks to form a single transmission. These chunks of spectrum can be non-contiguously aligned in frequency. This type of agile radios is often implemented in the form of OFDMA, requiring sophisticated signal processing techniques beyond those used in WiFi radios [5], [6], [7], [8], [9]. Our recent work has developed a fully decentralized $k$-agile radio [9]. The level of frequency-agility increases with $k$, so does the hardware complexity.

Intuitively, $k$-agile radios are more advantageous than 1agile radios in terms of spectrum access. This is particularly true when transmissions carry highly dynamic spectrum demands and experience heterogeneous interference conditions. In these cases, 1-agile radios, which must use a contiguous frequency chunk, face the problem of spectrum fragmentation. Over time, as individual transmissions adjust their spectrum usage, available spectrum becomes increasingly partitioned into a collection of discrete fragments. This fragmentation means that a significant portion of spectrum, while free, is effectively unusable because individual fragments do not provide the minimum contiguous frequency usage required by subsequent spectrum demands. $k$-agile radios, on the other hand, successfully suppress the impact of spectrum fragmentation directly at the physical layer. By utilizing multiple spectrum segments in a single transmission, each radio can now support high bandwidth transmissions even in the presence of spectrum fragmentation.

Ideally, one would prefer agile radios with large $k$ to obtain desired spectrum usage and suppress fragmentation. Aside from having higher implementation cost [9], larger $k$ also means higher spectrum overhead from "frequency guard 
bands" placed at allocated frequency boundaries. In distributed spectrum sharing, guard bands are required to suppress outof-band emissions that produce cross-band interference between links using different frequency chunks [10], [11]. Like those used by WiFi channels, guard bands are not usable for communication, thus are spectrum overhead and increase as transmissions make use of more frequency fragments. To effectively control the guard band overhead, a system would prefer agile radios with smaller $k$, because the overhead is at most $k$ times of those using 1 -agile radios.

From the above, we see that the choice of frequency-agile radios has clear implications on both network performance and hardware complexity. It is important to identify whether and when having additional frequency-agility matters and how much improvement it can lead to. In this paper, we approach this problem by asking and addressing the following question:

"In the context of dynamic spectrum sharing, how much performance improvement can $k$-agile radios achieve over 1 agile radios, and how would the choice of $k$ affect the results?"

We answer this question by considering a representative spectrum sharing scenario where multiple access points (or base stations) access and share spectrum to connect their subscribers. Using both theoretical analysis and simulation experiments, we examine and compare the amount of spectrum required to satisfy all access points' spectrum demands using 1 -agile and $k$-agile radios. For a fair comparison, we examine both worst case and average case performance to abstract the impact of spectrum demand and topology configuration. We also consider a complementary scenario where the total spectrum is given, and examine how often an access point cannot obtain its desired spectrum. To make the analysis tractable, we do not consider the impact of guard bands in our analytical results. Instead, we assume that the overhead is controlled by limiting $k$, and use simulation experiments to examine the impact of guard bands and verify our analytical claims.

To the best of our knowledge, this work is the first to provide a detailed comparison of 1 -agile and $k$-agile radios in distributed dynamic spectrum access. Our analytical and experimental results lead to the following interesting findings:

- In the worst case, using fully-agile radios $(k>>1)$ leads to arbitrarily large improvement over 1 -agile radios. The improvement becomes upper-bounded by $k$ when using $k$-agile radios.

- In the average case, the improvement is $10 \%-40 \%$ for typical traffic patterns and interference conditions. The benefit of frequency-agility increases when both demand dynamics and interference heterogeneity are present.

- In the average cases, 2-agile radios achieve the majority of improvement brought by fully-agile radios.

- Using the application-level disruption rate as an alternative performance metric, we found that increasing frequency-agility produces more visible impact to application-level performance.

\section{Preliminaries}

We consider a representative dynamic spectrum access scenario where access points or base stations (hereby referred to as nodes) share a spectrum band to support their subscribers. Due to user mobility and traffic dynamics, each node experiences time-varying traffic load and seeks to match spectrum usage to its traffic demand. Each node and its associated users are equipped with a single agile radio (1-agile or $k$-agile). The spectrum band is finely partitioned into a large set of frequency segments, where a segment refers to the minimum unit of spectrum usage. For example, when implemented via OFDMA [5], [9], each segment corresponds to a frequency subcarrier or a set of contiguously aligned subcarriers. We assume that the number of segments is large, e.g. 2562048. We also consider a distributed network setting where nodes access spectrum without any central control. For a fair comparison, we do not consider any primary users, because their presence by taking a chunk of the spectrum automatically imply that $k$-agile radios are advantageous. Instead, we focus on flat spectrum sharing among nodes.

We use the notion of $k$-agile radio to define radios with different levels of agility. A $k$-agile radio is able to use up to $k$ noncontiguous frequency chunks simultaneously, where each chunk is one or multiple contiguously aligned segments. We refer to radios that can access any combination of the segments as fully-agile radios. Thus, if the number of segments is $M$, a full-agile radio is a $k$-agile radio with $k=M / 2$. For easy notation, we refer to this fully-agile radio as $k=\infty$. Throughout the paper, we use the term chunk to refer to a set of contiguously aligned frequency segments/subcarriers. Thus a 1-agile radio is only able to access one chuck.

\section{Why Additional Agility Matters}

Before presenting our analysis, we first identify when and why having different levels of frequency-agility matters. we show that two inherent properties of dynamic spectrum access, namely spectrum demand dynamics and conflict topology heterogeneity, lead to the problem of spectrum fragmentation. The fragmentation is particularly harmful among 1 -agile radios, but can be suppressed effectively using $k$-agile radios. While prior work has observed the fragmentation problem [2], [12], [13], in this paper we formally model and analyze its penalty.

Spectrum Demand Dynamics. Adapting spectrum usage to time-varying demands is the key new feature of dynamic spectrum access. The nature of dynamic access, however, also leads to the problem of spectrum fragmentation. As individual nodes dynamically occupy, change and release their spectrum usages, the available spectrum becomes gradually divided into a set of individual fragments. Such fragmentation is particularly harmful for nodes using 1-agile radios. Many fragments, although free, are now unusable because they are too small to hold any subsequent demands requiring contiguous frequency. Nodes with $k$-agile radios, by combining multiple fragments into a single transmission, can maintain high-throughput transmissions even in the presence of spectrum fragmentation. 


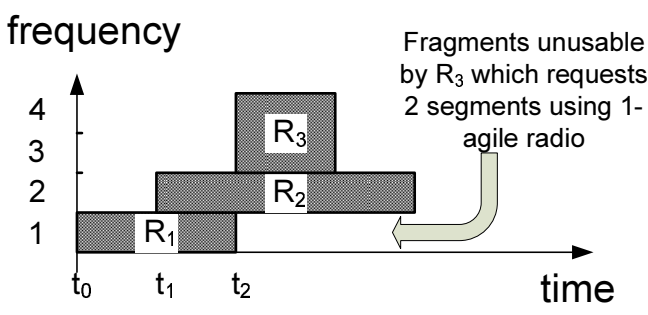

Fig. 2. An example of fragmentation due to dynamic spectrum demands. Because of the fragmentation, the total frequency consumption is 4 segments for 1-agile radios, and it reduces to 3 segments for 2 -agile radios.

Consider an example in Fig. 2 where three nodes dynamically acquire spectrum based on their present demands, each using a 1-agile radio. After obtaining its desired spectrum, each node maintains the same frequency usage till the end of its session or when its demand changes. In this example, when $R_{3}$ arrives and requests 2 (contiguous) frequency segments, it has to use segments $3-4$ although segment 1 is available. By using a 2 -agile radio, $R_{3}$ can combine segment 1 and 3 together, reducing the total frequency consumption from 4 to 3 segments. This example shows that using $k$-agile radios can effectively suppress spectrum fragmentation directly at the physical layer.

The fragmentation problem of 1-agile radios could be partially remedied using higher-layer solutions, although at a cost. The first solution optimizes spectrum allocation algorithms to reduce fragmentation. In the above example, if $R_{2}$ had occupied segment 3 rather than $2, R_{3}$ would have used segments 1 and 2. This solution, however, requires knowledge of future network-wide spectrum demands, which is generally infeasible in distributed networks that our paper targets. Indeed, we will show that no online algorithm can avoid a heavy penalty of fragmentation in the worst case. The second solution makes nodes "defragment" frequency on-the-fly, e.g. $R_{2}$ moves to segment 1 when $R_{1}$ departs. This solution, however, could lead to disruptions to ongoing transmissions as nodes defragment. The third solution is to compensate the frequency-domain fragmentation in the time-domain [14]. That is, nodes use less frequency than they originally desire but occupy a longer time period. In the above example, $R_{3}$ can now use 1 segment rather than 2 by taking twice the time to transmit its data. While this solution may work well for delay-tolerant applications like file transfer, it would lead to undesirable delay for realtime applications and ultimately visible disruptions to user applications.

While higher-layer solutions could partially remedy the fragmentation problem, they all come at some cost, either requiring heavy protocol complexity/overhead or resulting in application-level artifacts. Therefore, it is worthwhile to consider the physical layer solution of migrating to $k$-agile radios. In our analysis, we seek to quantify the advantage of such migration in the context of distributed spectrum access without time compensation and online defragmentation.

Conflict Topology Heterogeneity. Even without demand dynamics, spectrum fragmentation can still occur because

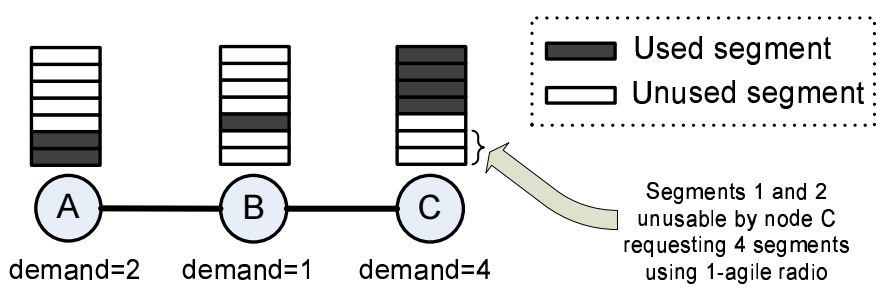

Fig. 3. An example of spectrum fragmentation due to network conflict topology. The total frequency consumption is 7 segments when using 1-agile radios, but reduces to 5 segments when using 2 -agile radios.

nodes experience heterogeneous interference conditions. Fig. 3 illustrates this fact using a simple example. Consider three nodes $A, B$ and $C$, each represented by a vertex in the figure. Two nodes are connected if they conflict with each other and cannot use the same frequency segment simultaneously. The blocks next to each vertex represent the available frequency segments, and the filled blocks are the segments assigned to the vertex. In this example, node $A$ has a demand of two segments and occupies segment 1 and 2 , and node $B$ demands one and occupies segment 3 to avoid conflicting with $A$. As a result, the usable spectrum for node $C$ is fragmented into two chunks: segments 1-2 and segments $4-7$. Using 1 -agile radios, $C$ has to use segments $4-7$, making the total frequency consumption of 7 segments for the system. On the other hand, using 2-agile radios, the frequency consumption is reduced to 5 .

Similarly, we now explore alternative solutions to suppress this fragmentation problem. For the example in Fig. 3, the fragmentation can be avoided using a different allocation: assigning segments 2 and 3 to node $A$, segment 1 to $B$ and segments $2-5$ to $C$. Thus, an interesting question is whether using 1-agile radios is good enough if one can optimize the allocation algorithm. Our analysis in Sec. VI, however, shows that even optimal algorithms cannot eliminate spectrum fragmentation in the presence of heterogeneous conflict conditions.

Frequency Guard Bands. From the above, we see that ideally one would prefer agile radios with large $k$ to fully overcome spectrum fragmentation and obtain desired frequency usage. In practice, however, larger $k$ also means higher spectrum overhead due to "frequency guard bands" placed at allocated frequency boundaries. Guard bands are required in distributed spectrum sharing to eliminate crossband interference from out-of-band emissions [10]. Out-ofband emissions are triggered by signal time-domain truncation and transmission nonlinearity [11]. These leakages can be controlled through advanced hardware design at the cost of higher hardware complexity and cost. By placing guard bands across frequency allocation boundaries, the degree of crossband interference can be effectively controlled.

Because guard bands are not usable for communication, they are essentially spectrum overhead and increase as transmissions make use of more frequency fragments. Guard bands are commonly used in existing distributed systems - each WiFi channel contains a set of guard bands at each side of its frequency boundary. Their sizes are related to constraints in 
hardware, processing and energy costs. To effectively control the guard band overhead, a system would prefer agile radios with smaller $k$, because the overhead is at most $k$ times of those using 1-agile radios.

\section{Methodology}

After identifying the benefit and cost of using $k$-agile radios, we now present our methodology for quantifying their gain over 1-agile radios. We start with the assumptions, then define our main metrics.

\section{A. Assumptions and Notations}

To make the analysis tractable, we introduce the following four assumptions.

First, we use an abstract model of conflict-free frequency access. By making spectrum usage conflict-free, nodes can use their desirable MAC access strategy independent of others. We assume that the interference constraints among nodes are represented into a static conflict graph. An example conflict graph is shown in Fig. 3 where two nodes are connected if they conflict with each other and cannot use the same frequency segment concurrently. We use this simple abstraction to make the analysis easy to explain, and refer the readers to recent work on deriving accurate conflict graphs from physical interference measurements [15]. Our analysis and conclusions can also be extended to other advanced interference models following the same methodology.

Second, focusing on spectrum access, our analysis assumes that all frequency segments are "homogeneous." They provide the same average transmission bandwidth and experience statistically the same interference conditions. In cases where segments are "heterogeneous," $k$-agile radios become even more advantageous by offering more freedom for nodes to choose segments. Our current analysis, however, does not consider this impact.

Third, to make the analysis tractable, we do not consider the impact of guard bands directly, and assume they are of negligible size compared to nodes' average spectrum demands and allocations. Instead, we indirectly examine their impact by analyzing $k$-agile radios with general $k$. We also verify the impact of guard bands using simulations.

Finally, we do not consider primary users in our analysis. The presence of primary users will further increase the need for $k$-agile radios. As primary users create fragments in available spectrum, secondary users with $k$-agile radios can scavenge multiple small fragments to form high-throughput transmissions, and even reduce their sensing overhead [16]. In this paper, however, we focus on dynamic spectrum sharing among cognitive radios without any primary users.

With the above assumptions in mind, we now define the notations used in our analysis. Consider the scenario described in Sec. II, where $N$ nodes request frequency segments in a distributed manner.

- Let $\mathbb{S}$ represent the traffic demand of the system. In general, $\mathbb{S}=\left\{R_{1}, R_{2}, \cdots\right\}$ where each $R_{i}$ is a request. A request $R_{i}$ is a 4-tuple that records the requesting node, the amount of frequency, the start time and the end time. For instance, in Fig. $2, \mathbb{S}=\left\{R_{1}, R_{2}, R_{3}\right\} . R_{1}$ starts at time $t_{0}$ and ends at $t_{2}$, requesting for 1 segment. In static traffic scenarios, $\mathbb{S}$ can be abbreviated using a vector of the spectrum demand of each node. For instance, in Fig $3, \mathbb{S}=\{2,1,4\}$.

- Let $a_{m, n, t}=1$ represent that the frequency segment $m$ is assigned to node $n$ at time $t$, and $a_{m, n, t}=0$ otherwise.

- Let $\mathbb{G}$ represent the conflict graph that captures the interference constraints among nodes. We require that at any time, an allocation is conflict-free, i.e. $a_{m, n, t} \cdot a_{m, k, t}=0$ if nodes $n$ and $k$ are connected in $\mathbb{G}$.

- Following the discussions in Sec. III, we measure the system spectrum efficiency using $\Omega_{k}\left(\mathbb{S}, \mathbb{A}_{k}, \mathbb{G}\right)$, the total number of frequency segments required to satisfy demand $\mathbb{S}$ using $k$ agile radios. It is evaluated under a conflict topology $\mathbb{G}$ using an allocation algorithm $\mathbb{A}_{k}$.

\section{B. Scenarios and Metrics}

We measure the performance distance of $k$-agile radios over 1-agile radios using

$$
\lambda_{k, 1}\left(\mathbb{S}, \mathbb{A}_{k}, \mathbb{A}_{1}, \mathbb{G}\right) \triangleq \frac{\Omega_{1}\left(\mathbb{S}, \mathbb{A}_{1}, \mathbb{G}\right)}{\Omega_{k}\left(\mathbb{S}, \mathbb{A}_{k}, \mathbb{G}\right)} .
$$

To understand the impact of dynamic traffic and conflict topology individually, we consider the following two scenarios:

Dynamic Traffic Scenario. Focusing on demand dynamics, we exclude the impact of conflict topology by assuming that all nodes conflict with each other. In this case, we abbreviate the notation

$$
\Omega_{k}\left(\mathbb{S}, \mathbb{A}_{k}\right) \triangleq \Omega_{k}\left(\mathbb{S}, \mathbb{A}_{k}, \mathbb{G}_{o}\right),
$$

where $\mathbb{G}_{O}$ is a clique graph. Accordingly,

$$
\lambda_{k, 1}\left(\mathbb{S}, \mathbb{A}_{k}, \mathbb{A}_{1}\right) \triangleq \frac{\Omega_{1}\left(\mathbb{S}, \mathbb{A}_{1}\right)}{\Omega_{k}\left(\mathbb{S}, \mathbb{A}_{k}\right)} .
$$

The example of Fig. 2 maps to the dynamic traffic scenario, and we have $\Omega_{1}\left(\mathbb{S}, \mathbb{A}_{1}\right)=4$ and $\Omega_{k}\left(\mathbb{S}, \mathbb{A}_{k}\right)=3,(k \geq 2)$. In this example $\mathbb{A}_{1}$ and $\mathbb{A}_{k}$ are commonly used FirstFit algorithms [17], which allocate lowest indexed spectrum chunks when possible. And we have $\lambda_{k, 1}\left(\mathbb{S}, \mathbb{A}_{k}, \mathbb{A}_{1}\right)=4 / 3(k \geq 2)$, mapping to a $33 \%$ improvement.

Clearly $\lambda_{k, 1}($.$) depends heavily on the spectrum demand$ $\mathbb{S}$ and the allocation algorithms. To abstract their impact, we consider the following two complementary metrics:

\section{1) Worst Case Distance:}

To measure the worst case $\lambda_{k, 1}($.$) , one possible option$ is to define a metric assuming both systems use the optimal algorithms $\mathbb{A}_{k}$ and $\mathbb{A}_{1}$. This option, however, assumes that the optimal algorithm is selected after knowing the traffic $\mathbb{S}$, and indeed measures the offline performance of the system. As we discussed in Fig. 2, since traffic prediction is in general very difficult, a more fair comparison would be to consider the online 
performance, i.e. the performance under all possible traffics for fixed algorithms:

$\lambda_{k, 1}^{\max }\left(\mathbb{A}_{k}, \mathbb{A}_{1}\right) \triangleq \max _{\mathbb{S}} \lambda_{k, 1}\left(\mathbb{S}, \mathbb{A}_{k}, \mathbb{A}_{1}\right)=\max _{\mathbb{S}} \frac{\Omega_{1}\left(\mathbb{S}, \mathbb{A}_{1}\right)}{\Omega_{k}\left(\mathbb{S}, \mathbb{A}_{k}\right)}$,

which is a function of algorithms $\mathbb{A}_{k}$ and $\mathbb{A}_{1}$.

\section{2) Average Case Distance:}

We also analyze the average case distance over all possible traffics $\mathbb{S}$ :

$$
\lambda_{k, 1}^{\text {mean }}\left(\mathbb{A}_{k}, \mathbb{A}_{1}\right) \triangleq \frac{\mathbf{E}_{\mathbb{S}}\left[\Omega_{1}\left(\mathbb{S}, \mathbb{A}_{1}\right)\right]}{\mathbf{E}_{\mathbb{S}}\left[\Omega_{k}\left(\mathbb{S}, \mathbb{A}_{k}\right)\right]},
$$

which is also a function of algorithms $\mathbb{A}_{k}$ and $\mathbb{A}_{1}$.

Heterogeneous Conflict Topology Scenario In this scenario, we consider static traffics and exclude the impact of demand dynamics. Because the demand is static and given, one can determine an optimal allocation that minimizes the frequency consumption. Thus we define

$$
\Omega_{k}(\mathbb{S}, \mathbb{G}) \triangleq \min _{\mathbb{A}_{k}} \Omega_{k}\left(\mathbb{S}, \mathbb{A}_{k}, \mathbb{G}\right),
$$

as the amount of frequency segments required using an optimal allocation algorithm based on $\mathbb{S}$. To abstract the impact of $\mathbb{S}$ and $\mathbb{G}$, we consider the following two complementary metrics:

1) Worst Case Distance:

$$
\lambda_{k, 1}^{\max } \triangleq \max _{\mathbb{S}, \mathbb{G}} \lambda_{k, 1}(\mathbb{S}, \mathbb{G})=\max _{\mathbb{S}, \mathbb{G}} \frac{\Omega_{1}(\mathbb{S}, \mathbb{G})}{\Omega_{k}(\mathbb{S}, \mathbb{G})} .
$$

By considering optimal allocation algorithms for both radios, we maintain a fair comparison between the two.

\section{2) Average Case Distance:}

$$
\lambda_{k, 1}^{\text {mean }} \triangleq \frac{\mathbf{E}_{\mathbb{S}, \mathbb{G}}\left[\Omega_{1}(\mathbb{S}, \mathbb{G})\right]}{\mathbf{E}_{\mathbb{S}, \mathbb{G}}\left[\Omega_{k}(\mathbb{S}, \mathbb{G})\right]}
$$

In the following two sections, we analyze $\lambda_{k, 1}(\cdot)$ for the two scenarios, respectively.

\section{IMPACT OF DEMAND DYNAMICS}

In this section, we analyze the impact of frequency agility under dynamic spectrum demands. To independently study the impact of demand dynamics, we assume the nodes are in a single conflict domain, i.e. $\mathbb{G}$ is always a clique. As shown in Fig. 2, radio agility is beneficial when the available spectrum is fragmented into separate chunks. This is analogous to the fragmentation problem in memory allocation [18], although previous works on memory allocation only consider $k=1$ and $k=\infty$. While our analysis is inspired by these previous results, a major difference is that we indirectly capture the impact of the guard band overhead by exploring the cases for small $k=2,3,4, \cdots$.

\section{Summary of Results}

- In the worst case, fully-agile radios have an arbitrarily large improvement over 1-agile radios, regardless of the on-line allocation algorithm that 1 -agile radios use.
- In the worst case, 2-agile radios have an improvement ratio between 1.5 and 2 over 1 -agile radios.

- In the average case, $k$-agile radios have an improvement ratio of $3 x_{k} /\left(2 x_{k}+1\right)$ over 1 -agile radios, where $x_{k}$ is the expected number of chunks used by each request.

- Simulation results show that our analysis satisfactorily predicts the average performance of $k$-agile radios. Using $k$-agile radios reduces spectrum consumption by up to $22 \%$ over 1 -agile radios. Noticeably, 2 -agile radios achieve most of the improvement.

\section{A. Worst Case Distance}

We first consider the extreme case $\lambda_{\infty, 1}^{\max }\left(\mathbb{A}_{\infty}, \mathbb{A}_{1}\right)$ (Eq. 1). It is easy to see that for fully-agile radios, the optimal algorithm $\mathbb{A}_{\infty}$ selects the lowest indexed segments when possible, regardless of the demand. Thus we focus on examining the impact of $\mathbb{A}_{1}$ :

Theorem $1 \lambda_{\infty, 1}^{\max }\left(\mathbb{A}_{\infty}, \mathbb{A}_{1}\right)$ is unbounded regardless of $\mathbb{A}_{1}$. Namely, for any allocation algorithm that 1-agile radios use, there exists a dynamic traffic $\mathbb{S}$ such that $\lambda_{\infty, 1}^{\max }\left(\mathbb{A}_{\infty}, \mathbb{A}_{1}\right)$ is arbitrarily large.

Proof: The theorem follows directly from a result in [19]. It shows that for any online allocation algorithm using contiguous memory allocation, the total memory required to satisfy a set of dynamic requests $\mathbb{S}$ could be at least $r \cdot \omega(\mathbb{S})$. Here $\omega(\mathbb{S})$ is the maximum total size of all active requests at any given time. $r$ is a factor that scales logarithmically with the maximum size of a request, and is thus unbounded.

The above result implies $\Omega_{1}\left(\mathbb{S}, \mathbb{A}_{1}\right) \geq r \cdot \omega(\mathbb{S})$ in the context of spectrum allocation. It is easy to see that $\Omega_{\infty}\left(\mathbb{S}, \mathbb{A}_{\infty}\right)=$ $\omega(\mathbb{S})$. Therefore, $\lambda_{\infty, 1}^{\max }\left(\mathbb{A}_{\infty}, \mathbb{A}_{1}\right) \geq r$ is unbounded in the worst case.

Now we turn to $\lambda_{k, 1}^{\max }\left(\mathbb{A}_{k}, \mathbb{A}_{1}\right)$ when $k$ is finite. First we prove an upper bound:

Theorem 2 For any $\mathbb{A}_{k}$, there exists an $\mathbb{A}_{1}$ such that $\lambda_{k, 1}^{\max }$ $\left(\mathbb{A}_{k}, \mathbb{A}_{1}\right) \leq k, k=2,3,4, \cdots$. Namely, 1-agile radios can support any traffic $\mathbb{S}$, using at most $k$ times the spectrum that $k$-agile radios use.

Proof: We design a strategy for 1-agile radios to select frequency segments, such that for any traffic, they use no more than $k$ times the spectrum consumed by $k$-agile radios.

In this strategy, we first allocate spectrum assuming nodes have $k$-agile radios. Each request can receive multiple chunks but at least one chunk is no less than $1 / k$ of the request size. Next, for each request we "inflate" its assigned chunks by $k$-folds in frequency so that the largest one is large enough to support the entire request. Finally, we "reassign" each request to its largest inflated chunk so that all the requests are supported using 1-agile radios. In this way, we have derived an allocation strategy for nodes with 1-agile radios, and the corresponding spectrum consumption is of $k$ times that from using $k$-agile radios. Fig. 4 illustrates the concept using a 

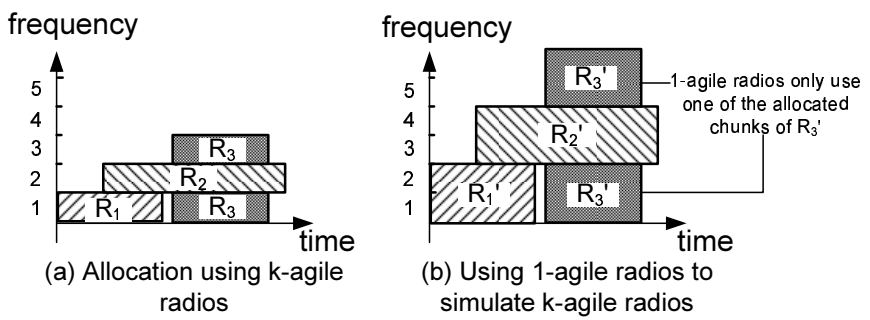

Fig. 4. Using 1-agile radios to simulate $k$-agile radios, using $k$ times the spectrum that $k$-agile radios use. The figure shows a case when $k=2$. In b), the 1-agile radio only utilizes one of the two allocated (grey) chunks to support demand $R_{3}$.

simple example assuming $k=2$ where three nodes using 1 -agile radios consume twice the spectrum.

Next, we show a lower bound for $k=2$ :

Theorem 3 Using a simple algorithm $\mathbb{A}_{2}$, for any $\mathbb{A}_{1}$, $\lambda_{2,1}^{\max }\left(\mathbb{A}_{2}, \mathbb{A}_{1}\right) \geq 1.5$. Namely, regardless of the allocation algorithm that 1 -agile radios use, 2-agile radios can achieve an improvement ratio of at least 1.5 over 1-agile radios under certain dynamic traffic $\mathbb{S}$.

Proof: To prove the theorem, we focus on a special type of traffic $\mathbb{S}^{+}$, where all requests have sizes of 1 or 2 segments. It is obvious that for this type of traffic, 2-agile radios can fully utilize the spectrum by using a simple (e.g. FirstFit) algorithm $\mathbb{A}_{2}$. Thus, $\Omega_{2}\left(\mathbb{S}^{+}, \mathbb{A}_{2}\right)$ equals $\omega\left(\mathbb{S}^{+}\right)$, the maximum sum of all the active requests in $\mathbb{S}^{+}$at any given time.

In [19], it is shown that $\forall \mathbb{A}_{1}, \Omega_{1}\left(\mathbb{S}^{+}, \mathbb{A}_{1}\right) \geq\left(3 \omega\left(\mathbb{S}^{+}\right)-\right.$ $1) / 2$, for some traffic $\mathbb{S}^{+}$that depends on $\mathbb{A}_{1}$. Thus we have

$$
\lambda_{2,1}^{\max }\left(\mathbb{A}_{2}, \mathbb{A}_{1}\right)=\max _{\mathbb{S}^{+}} \frac{\Omega_{1}\left(\mathbb{S}^{+}, \mathbb{A}_{1}\right)}{\omega\left(\mathbb{S}^{+}\right)} \geq \frac{\left(3 \omega\left(\mathbb{S}^{+}\right)-1\right) / 2}{\omega\left(\mathbb{S}^{+}\right)} .
$$

Taking the limit $\omega\left(\mathbb{S}^{+}\right) \rightarrow \infty$, we get $\lambda_{2,1}^{\max }\left(\mathbb{A}_{2}, \mathbb{A}_{1}\right) \geq 1.5$.

An example where $\frac{\Omega_{1}\left(\mathbb{S}^{+}, \mathbb{A}_{1}\right)}{\omega\left(\mathbb{S}^{+}\right)}=1.5$ is shown in Fig. 5, where $\mathbb{A}_{1}$ is a FirstFit algorithm [17] that allocates lowest indexed segments when possible. Initially the system contains eight nodes each occupying one frequency segment, thus segments 1-8 are occupied. Then four of the eight requests depart, releasing four frequency fragments of size 1 segment. When two new requests (each asking for 2 segments) arrive, they cannot use the fragments when equipped with 1 -agile radios. In this case, $\Omega_{1}\left(\mathbb{S}^{+}, \mathbb{A}_{1}\right)=12$. It is clear that $\omega\left(\mathbb{S}^{+}\right)=8$ and thus $\frac{\Omega_{1}\left(\mathbb{S}^{+}, \mathbb{A}_{1}\right)}{\omega\left(\mathbb{S}^{+}\right)}=1.5$. Note that in this example we consider a specific algorithm $\mathbb{A}_{1}$. For other algorithms, the traffic $\mathbb{S}^{+}$that creates the worst case performance for 1-agile radios might change, but the intuition remains the same: creating a large number of fragments each of size 1 segment.

Together, Theorems 2 and 3 can be interpreted into the following corollary:

Corollary 1 The improvement ratio of 2-agile radios over 1 agile radios is up to $r$, where $1.5 \leq r \leq 2$.

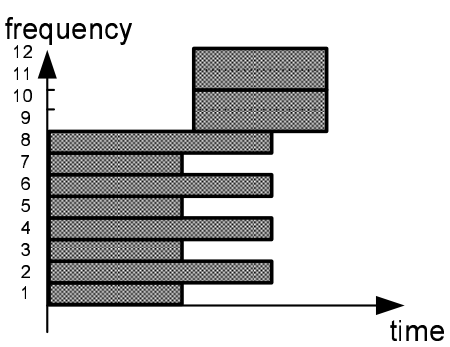

Fig. 5. A case where $\frac{\Omega_{1}\left(\mathbb{S}^{+}, \mathbb{A}_{1}\right)}{\omega\left(\mathbb{S}^{+}\right)}=1.5$. All requests have sizes of 1 or 2 segments. The traffic dynamics create fragmented segments $(1,3,5$, and 7$)$ that are not usable by subsequent requests. As a result, 1-agile radios require 12 segments while 2 -agile radios require only 8 segments.

Theorems 1 and 2 show that in terms of the worst case distance, the benefit of $k$-agile radios is bounded while the benefit of fully-agile radios is unbounded. This result implies that highly-agile radios have the potential to significantly improve spectrum usage efficiency. It also motivates us to investigate the benefit further from the average case viewpoint.

\section{B. Average Case Distance}

In the following, we study the average case distance by averaging over the traffic pattern $\mathbb{S}: \lambda_{k, 1}^{\text {mean }}\left(\mathbb{A}_{k}, \mathbb{A}_{1}\right) \triangleq \frac{\mathbf{E}_{\mathbb{S}}\left[\Omega_{1}\left(\mathbb{S}, \mathbb{A}_{1}\right)\right]}{\mathbf{E}_{\mathbb{S}}\left[\Omega_{k}\left(\mathbb{S}, \mathbb{A}_{k}\right)\right]}$ (Eq. 2). Clearly the results depend on the traffic model. Advanced traffic models from recent measurement results [20], however, are highly complex and make the analysis intractable. Thus, we focus on a simple and yet general model in our theoretical analysis, and in Sec. V-C use network simulations to verify our analysis and study the impact of traffic statistics.

For our theoretical analysis, we focus on a specific type of traffic model where the traffic stabilizes after a period of time. Our analytical model is inspired by a result in memory allocation, referred to as the fifty percent rule [17]. It states that: "In a dynamic memory allocation system, using a simple FirstFit allocation algorithm, the expected utilization that the system can stably support is $2 / 3$."

Inspired by this model, we make the following assumptions in our analysis. We assume that the traffic is recurrent and stabilizes after a period of time. This is a property found in many typical network applications. Using the FirstFit [17] algorithm for both $\mathbb{A}_{k}$ and $\mathbb{A}_{1}$, the system will reach an equilibrium state. At an equilibrium state, the following properties hold:

1) The number of allocated chunks and the number of empty chunks stabilize.

2) The average size of empty chunks is approximately equal to the average size of allocated chunks.

3) Using $k$-agile radios, each request is satisfied by an expected number of $x_{k}$ chunks $\left(x_{k} \leq k\right)$. The relationship between $x_{k}$ and $k$ depends on the traffic.

Based on the above assumptions, we estimate the expected spectrum consumption for $k$-agile radios:

Theorem $4 \mathbf{E}_{\mathbb{S}}\left[\Omega_{k}\left(\mathbb{S}, \mathbb{A}_{k}\right)\right]=\frac{2 x_{k}+1}{2 x_{k}} \mathbf{E}[\mathbb{S}], k=1,2,3, \cdots$. Here $\mathbf{E}[\mathbb{S}]$ is the expected total demand of all active requests when the system is in an equilibrium state. 


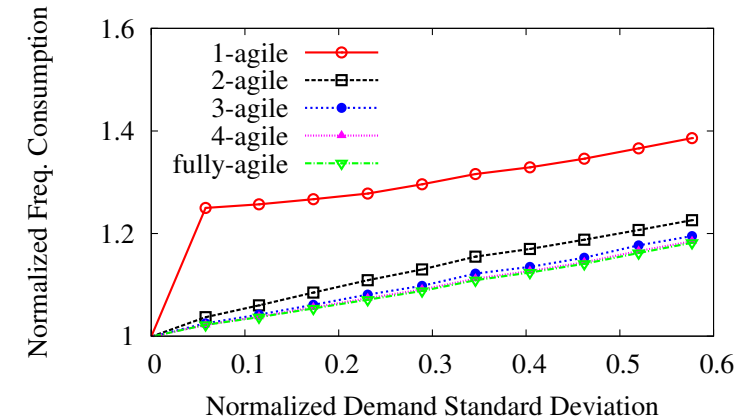

(a) Normalized frequency consumption, no guard band

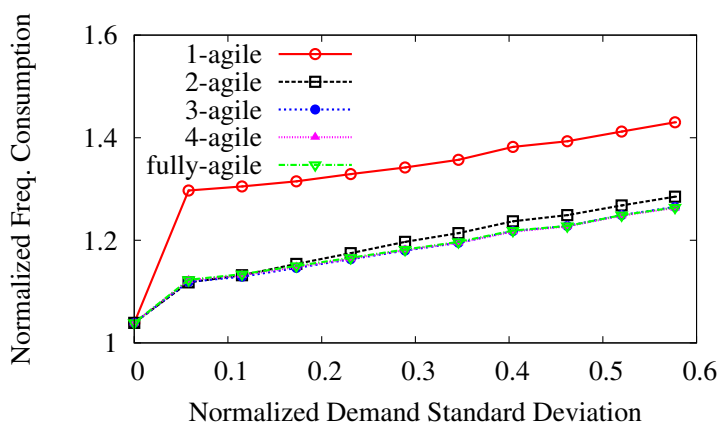

(c) Normalized frequency consumption, guard band size $=4$ segments per chunk.

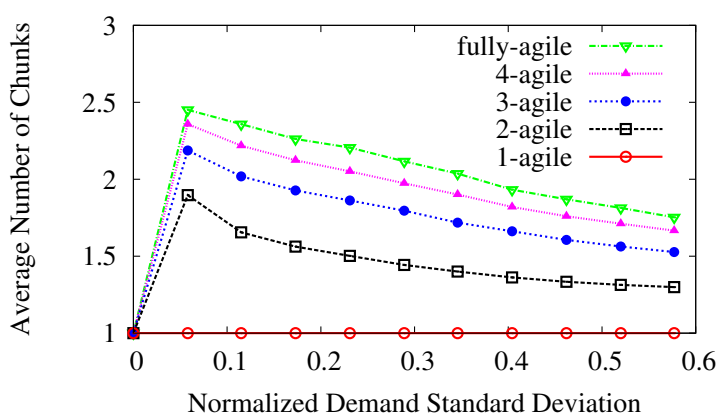

(b) Average number of chunks used by each request, no guard band

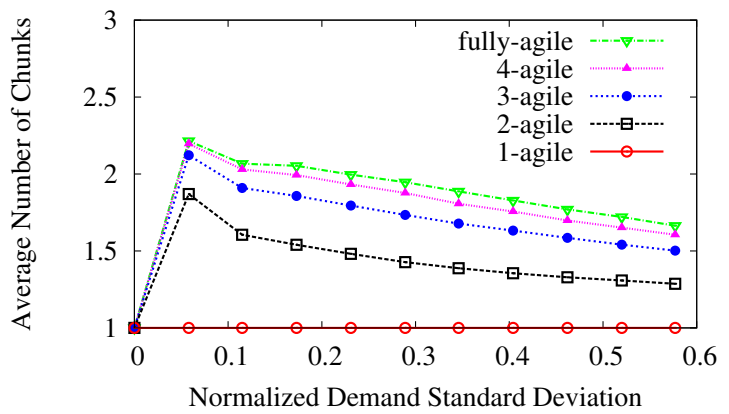

(d) Average number of chunks used by each request, guard band size $=4$ segments per chunk.

Fig. 6. The average case impact of radio agility under dynamic spectrum demands. We examine the normalized frequency consumption and the average number of chunks used by each request. The same experiment is repeated for guard band sizes of 0 and 4 segments, respectively. We also experimented under 30 and 50 nodes and obtained similar results.

The proof of Theorem 4 is in the Appendix.

Directly from Theorem 4 , we quantify the average case benefit of $k$-agile radios over 1 -agile radios:

Theorem 5 The average case distance $\lambda_{k, 1}^{\text {mean }}\left(\mathbb{A}_{k}, \mathbb{A}_{1}\right) \triangleq$ $\frac{\mathbf{E}_{\mathbb{S}}\left[\Omega_{1}\left(\mathbb{S}, \mathbb{A}_{1}\right)\right]}{\mathbf{E}_{\mathbb{S}}\left[\Omega_{k}\left(\mathbb{S}, \mathbb{A}_{k}\right)\right]}=\frac{3 x_{k}}{2 x_{k}+1}$.

Proof: Obviously $x_{1}=1$. So we have

$$
\frac{\mathbf{E}_{\mathbb{S}}\left[\Omega_{1}\left(\mathbb{S}, \mathbb{A}_{1}\right)\right]}{\mathbf{E}_{\mathbb{S}}\left[\Omega_{k}\left(\mathbb{S}, \mathbb{A}_{k}\right)\right]}=\frac{\frac{2 x_{1}+1}{2 x_{1}} \mathbf{E}[\mathbb{S}]}{\frac{2 x_{k}+1}{2 x_{k}} \mathbf{E}[\mathbb{S}]}=\frac{\frac{3}{2}}{\frac{2 x_{k}+1}{2 x_{k}}}=\frac{3 x_{k}}{2 x_{k}+1} .
$$

Theorem 5 implies that the average case improvement brought by $k$-agile radios is upper bounded by a factor of 1.5 (when $x_{k} \rightarrow \infty$ ). Compared to the results in Theorem 1 and 2, it is clear that radio agility has higher impact in the worst case performance than the average case.

We will compare the analytical results in Theorem 5 with simulation results in the following subsection.

\section{Simulation Results}

In this subsection, we use network simulations to verify and complement the analysis in Sec. V-B. We also study the impact of traffic statistics and guard bands.

We simulate 10 nodes sharing an arbitrarily large spectrum trunk and examine the total spectrum consumed $\Omega_{k}\left(\mathbb{S}, \mathbb{A}_{k}\right)$. To simulate dynamic demands, we configure each node to change its demand in exponentially distributed time intervals, and the volume of each demand is uniformly distributed with a mean value of 100 segments. To access spectrum, each node applies an online greedy algorithm to occupy unused spectrum chunks and minimize $\Omega_{k}\left(\mathbb{S}, \mathbb{A}_{k}\right)$ in its current view. For instance, consider an example where at time $t$ the first 500 segments are occupied, and at time $t+1$ two nodes depart and leave two frequency fragments of size 10 and 20 segments, respectively. At $t+2$ a new node demands 30 segments. Using a 1-agile radio, the node will occupy the 501th through 540 th segments, making $\Omega_{1}()=$.540 , while using a 2 -agile radio the node will combine the two fragments, leaving $\Omega_{2}()=$.500 .

Fig. 6(a) compares the system-wide spectrum consumption $\Omega_{k}\left(\mathbb{S}, \mathbb{A}_{k}\right)$ for different $k$. We normalize the $y$-axis by dividing $\Omega_{k}\left(\mathbb{S}, \mathbb{A}_{k}\right)$ by the average traffic load. To understand the impact of demand dynamics, we fix the mean demand volume and vary their standard deviations from 0 to 0.6 . We observe that the spectrum consumption does increase with the level of demand dynamics, but the differences among different radios remain stable. In this example configuration, 2-agile radios achieve a major improvement of $13 \%-20 \%$ over 1 -agile radios. As $k$ increases, the improvement converges quickly. Finally, fully-agile radios gain by $17 \%-22 \%$ over 1 -agile radios.

To validate our analytical results in Theorem 5, we first measure $x_{k}$, the expected number of chunks used by each request from the experiments. Results in Fig 6(b) show that $x_{k}$ decreases as the demand variance increases. This observation 
TABLE I

Comparing the AVERAGE CASE Distance $\lambda_{k, 1}^{\text {mean }}\left(\mathbb{A}_{k}, \mathbb{A}_{1}\right)$ DERIVED FROM OUR ANALYSIS AND SIMULATIONS.

\begin{tabular}{l|llll}
\hline & \multicolumn{4}{|c}{$k$-agile radio } \\
& $\mathrm{k}=\infty$ & $\mathrm{k}=4$ & $\mathrm{k}=3$ & $\mathrm{k}=2$ \\
\hline 10 nodes (Simulation) & 1.1726 & 1.1696 & 1.1598 & 1.1305 \\
\hline 10 nodes (Theorem 5) & 1.1671 & 1.1537 & 1.1300 & 1.0831 \\
\hline 30 nodes (Simulation) & 1.1525 & 1.1456 & 1.1348 & 1.1028 \\
\hline 50 nodes (Simulation) & 1.1296 & 1.1256 & 1.1160 & 1.0879 \\
\hline
\end{tabular}

is consistent with Theorem 4 , which predicts that $\Omega_{k}\left(\mathbb{S}, \mathbb{A}_{k}\right)$ increases as $x_{k}$ decreases. We select the results with traffic volume uniformly distributed in $[1,200]$ (i.e. with standard deviation 0.577) and compare the $\lambda_{k, 1}^{\text {mean }}\left(\mathbb{A}_{k}, \mathbb{A}_{1}\right)$ values obtained by two methods: the simulation results from Fig. 6(a) and the analytical results from Theorem 5 with $x_{k}$ measured from Fig. 6(b). Table I shows that the analytical results provide a reasonable prediction of the improvement. We also look at the impact of node density to the above results. Table I shows that the average gain is relatively stable when the number of nodes increases to 30 and 50 . It slightly decreases because of the higher level of multiplexing.

Finally, we examine the impact of guard band overhead on the above results. We repeat the simulations assuming a guard band size of 4 segments per frequency chunk, mapping to an average $4 \%$ overhead for 10 nodes using 1-agile radios. Fig. 6(c) and 6(d) show that while the spectrum consumption increases due to the guard band overhead, the improvement of $k$-agile radios decreases. In particular, the improvement of fully-agile radios reduces to $13 \%-15 \%$.

\section{IMPACT OF CONFLICT TOPOLOGY}

In this section, we study how conflict topology affects agile radios' spectrum usage. We consider a network where nodes are placed in a large space, and some of them do not conflict with each other. Instead, nodes have different conflicting peers and only need to avoid using the same segments occupied by conflicting peers. As we have shown in Sec. III, because nodes have different views of their spectrum availability, they will produce fragments in spectrum usage.

To focus solely on the impact of conflict topology, we assume the spectrum demands are static, reducing $\mathbb{S}$ to a vector recording the number of segments requested by each node. For instance, in Fig. 3, $\mathbb{S}=\{2,1,4\}$.

\section{Summary of Results}

- We show that the fragmentation problem caused by heterogeneous conflict topology is unavoidable even under optimal allocation algorithms.

- In the worst case, $\lambda_{\infty, 1}^{\max } \triangleq \max _{\mathbb{S}, \mathbb{G}} \lambda_{\infty, 1}(\mathbb{S}, \mathbb{G}$ ) (Eq. 3) is unbounded for general conflict topologies.

- For special conflict topologies, we interpret previous results in approximation algorithms and show that $\lambda_{\infty, 1}^{\max }$ is bounded by small constants for unit disk graphs and interval graphs.

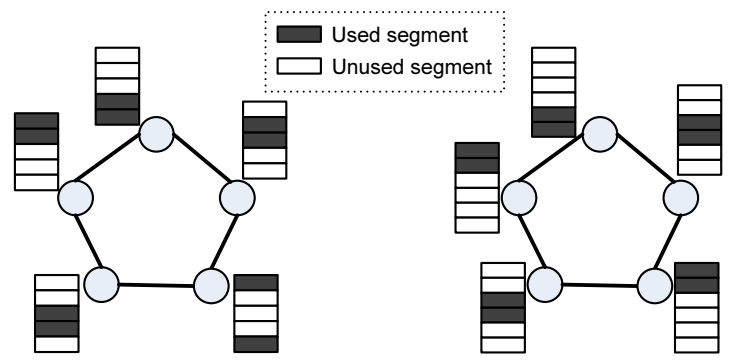

(a) $k$-agile radios $(k \geq 2)$ require 5 segments (b) 1-agile radios

Fig. 7. An example illustrating the benefit of frequency-agility under heterogeneous conflict topology. The network contains five nodes and their conflict graph is a ring topology. The filled blocks represent the segments used by each node. To assign each node 2 segments without any conflict, in (a) using $k$-agile radios $(k \geq 2)$ requires 5 segments, while in (b) using 1-agile radios requires 6 segments.

- Simulation results show that 2 -agile radios achieve 10 $12 \%$ improvement, and the additional improvement using a larger $k$ is very small.

\section{A. Worst Case Distance}

We start with the worst case analysis. As we mentioned in Sec. III, the fragmentation problem in the Fig. 3 example can be avoided with a better allocation. However, we find that in general cases, fragmentation is a fundamental artifact of the conflict topology and cannot be avoided by optimizing the allocation. Consider the example in Fig. 7, where 5 nodes form a ring conflict topology. Assume the traffic demand $\mathbb{S}=$ $\{2,2,2,2,2\}$. Using $k$-agile radios $(k \geq 2), 5$ segments are sufficient to satisfy the demand (Fig. 7(a)). In contrast, using 1-agile radios, at least 6 segments are required (Fig. 7(b)). In this case, $\frac{\Omega_{1}(\mathbb{S}, \mathbb{G})}{\Omega_{k}(\mathbb{S}, \mathbb{G})}=6 / 5$, leading to an $20 \%$ improvement.

Next, we generalize the above observation and examine $\lambda_{\infty, 1}^{\max } \triangleq \max _{\mathbb{S}, \mathbb{G}} \lambda_{\infty, 1}(\mathbb{S}, \mathbb{G})$. The answer depends on the topology $\mathbb{G}$, so we start with general conflict topologies.

General Conflict Topology We show that when there is no restriction on $\mathbb{G}, \lambda_{\infty, 1}^{\max }$ can be arbitrarily large.

Theorem $6 \lambda_{\infty, 1}^{\max }$ is unbounded. Namely, for any $c>1$, there exists a network topology $\mathbb{G}^{*}$ and traffic $\mathbb{S}^{*}$ such that $\frac{\Omega_{1}\left(\mathbb{S}^{*}, \mathbb{G}^{*}\right)}{\Omega_{\infty}\left(\mathbb{S}^{*}, \mathbb{G}^{*}\right)} \geq c$.

Proof: Our proof utilizes a result of graph coloring theory: the ratio between a graph's Chromatic Number and its Fractional Chromatic Number can be arbitrarily large [21]. In the following, we first define the chromatic number and the fractional chromatic number, and then prove the main result.

Given a graph $\mathbb{G}$, its Chromatic Number $\chi(\mathbb{G})$ is the minimum number of colors required to color all the vertices such that no two directly connected vertices share the same color. By its definition, $\chi(\mathbb{G})=\Omega_{1}(\{1,1, \cdots, 1\}, \mathbb{G})$. In the example of Fig. 7, $\chi(\mathbb{G})=3$. For the same graph $\mathbb{G}$, the Fractional Chromatic Number $\chi_{F}(\mathbb{G})$ is the minimum number of colors required if the color received by each 


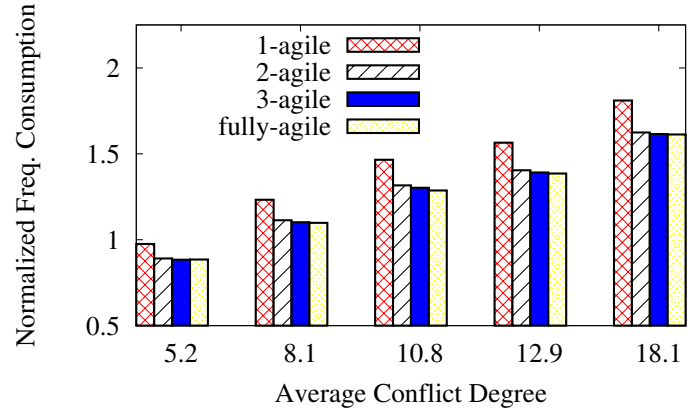

(a) Uniform Random Topology

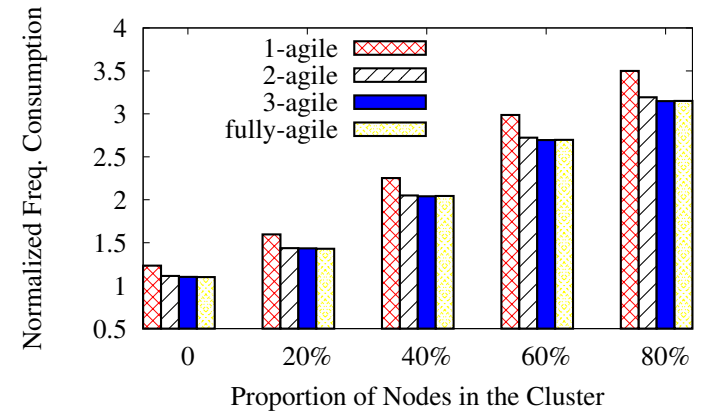

(b) Clustered Topology

Fig. 8. The average case impact of frequency agility under heterogeneous conflict topologies. In both uniform and clustered topologies, 2-agile radios reduce the spectrum consumption by $10 \%-12 \%$ compared to 1 -agile radios.

node can be divided into fractions and assigned in a noncontiguous manner. Like the example of Fig. 7(a), by mapping each segment into a $1 / 2$ color, we need 2.5 colors and thus $\chi_{F}(\mathbb{G})=2.5$.

Using these definitions, we show that the following result holds (The proof is listed in the Appendix):

Lemma 1 For any $\mathbb{G}$, there exists an integer $r \geq 1$,

$$
\begin{aligned}
\Omega_{\infty}(\{r, r, \cdots, r\}, \mathbb{G}) & =r \cdot \chi_{F}(\mathbb{G}) \\
\Omega_{1}(\{r, r, \cdots, r\}, \mathbb{G}) & =r \cdot \chi(\mathbb{G}) .
\end{aligned}
$$

For the example of Fig. 7, we have

$$
\begin{aligned}
\Omega_{\infty}(\{2,2, \cdots, 2\}, \mathbb{G}) & =5=2 \chi_{F}(\mathbb{G}) \\
\Omega_{1}(\{2,2, \cdots, 2\}, \mathbb{G}) & =6=2 \chi(\mathbb{G}) .
\end{aligned}
$$

In [21], it has been shown that for any $c>1$, there always exists a graph $\mathbb{G}^{*}$ where $\frac{\chi\left(\mathbb{G}^{*}\right)}{\chi_{F}\left(\mathbb{G}^{*}\right)} \geq c$. Using $\mathbb{G}^{*}$ and Lemma 1 , we can always find an $r$ and $\mathbb{S}^{*} \triangleq\{r, r, \cdots, r\}$, where $\frac{\Omega_{1}\left(\mathbb{S}^{*}, \mathbb{G}^{*}\right)}{\Omega_{\infty}\left(\mathbb{S}^{*}, \mathbb{G}^{*}\right)}=\frac{r \cdot \chi\left(\mathbb{G}^{*}\right)}{r \cdot \chi_{F}\left(\mathbb{G}^{*}\right)} \geq c$

For $\lambda_{k, 1}^{\max }$, we have the following upper bound. The proof uses a technique very similar to that of Theorem 2 , and is thus omitted due to space limitation.

Theorem $7 \lambda_{k, 1}^{\max } \leq k, k=2,3,4, \cdots$. Namely, 1-agile radios can always support any traffic $\mathbb{S}$, using at most $k$ times the amount of spectrum that $k$-agile radios use.

Special Conflict Topology While $\lambda_{\infty, 1}^{\max }$ is unbounded in general, the worst case happens only for some special conflict topologies [21]. For conflict topologies that are more likely to appear in real networks, however, $\lambda_{\infty, 1}^{\max }$ could be bounded. Now we consider two well-known graphs: unit-disk graphs and interval graphs. Unit-disk graphs map to the distance-based interference model where two access points conflict if they are located within a predefined distance. Interval graphs are the one-dimensional version of unit disk graphs. Both graphs have been widely used to model wireless interference conditions.

To upper-bound $\lambda_{\infty, 1}^{\max }$ for interval graphs and unit disk graphs, we interpret previous results in approximation algorithms. We define $\omega(\mathbb{S}, \mathbb{G})$ as the maximum total size of all requests in a clique of $\mathbb{G}$, which is a lower bound of $\Omega_{\infty}(\mathbb{S}, \mathbb{G})$, i.e. $\omega(\mathbb{S}, \mathbb{G}) \leq \Omega_{\infty}(\mathbb{S}, \mathbb{G})$. Prior work [22], [23] shows that $\Omega_{1}(\mathbb{S}, \mathbb{G}) \leq 7 \cdot \omega(\mathbb{S}, \mathbb{G})$ for unit disk graphs, and $\Omega_{1}(\mathbb{S}, \mathbb{G}) \leq 3 \cdot \omega(\mathbb{S}, \mathbb{G})$ for interval graphs. Thus we build the following theorem:

Theorem $8 \lambda_{\infty, 1}^{\max } \leq 7$ if the conflict topology is a unit disk graph, $\lambda_{\infty, 1}^{\max } \leq 3$ if the conflict topology is an interval graph.

Again, the worst case analytical results reveal the potential benefit of using more frequency-agility. To obtain a comprehensive view of this issue, we now investigate the average case performance.

\section{B. Average Case Distance}

In evaluating the average case benefit $\lambda_{k, 1}^{\text {mean }}$ (Eq. 4), we face two challenges. First, the benefit depends on the statistical distribution of the conflict graph $\mathbb{G}$, which is intractable to model since it involves hard combinatorial distributions. Thus, we rely on simulations to study $\lambda_{k, 1}^{\text {mean }}$. The second challenge is that given any $\mathbb{S}$ and $\mathbb{G}$, it is NP-hard to obtain the optimal allocations and determine $\Omega_{k}(\mathbb{S}, \mathbb{G}) \triangleq \min _{\mathbb{A}_{k}} \Omega_{k}\left(\mathbb{S}, \mathbb{A}_{k}, \mathbb{G}\right)$. We thus consider both $\mathbb{A}_{k}$ and $\mathbb{A}_{1}$ to be a greedy algorithm: sequentially allocate segments to satisfy nodes' traffic. Each node chooses segments greedily to minimize $\Omega_{k}\left(\mathbb{S}, \mathbb{A}_{k}, \mathbb{G}\right)$ in the current step. This is the same greedy strategy used in Sec. V-C.

We perform experiments using both random uniform and clustered conflict topologies. In the first set of experiments, we randomly place 100 nodes on a $1000 \mathrm{~m} \times 1000 \mathrm{~m}$ plane, and use a simple criteria to construct the conflict graph $\mathbb{G}$ : two nodes conflict if they are within distance $d$. For each node, we randomly generate a static traffic volume uniformly distributed in $[1,40]$. Fig. 8(a) evaluates $\lambda_{k, 1}^{\text {mean }}$ under different network densities by varying $d$. The $x$-axis lists the average conflict degree of the network, i.e. the per-node expected number of conflicting peers. Again we see that the majority of improvements is achieved by 2 -agile radios which reduce spectrum consumption by $10 \%-12 \%$.

Next we repeat the above experiment but using clustered topologies. To generate clustered topologies, we put a proportion of the nodes in a $300 m \times 400 m$ area and distribute 


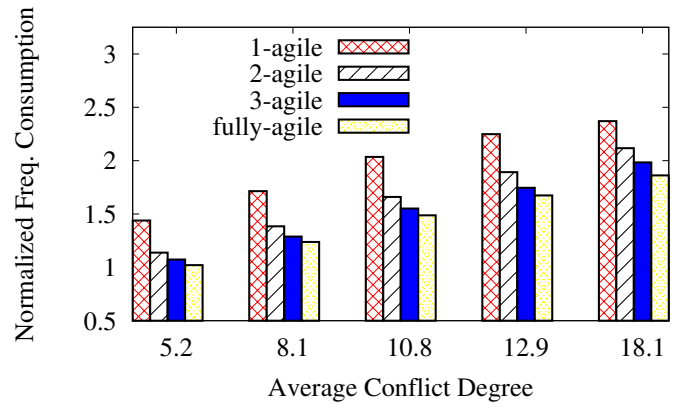

(a) No guard band

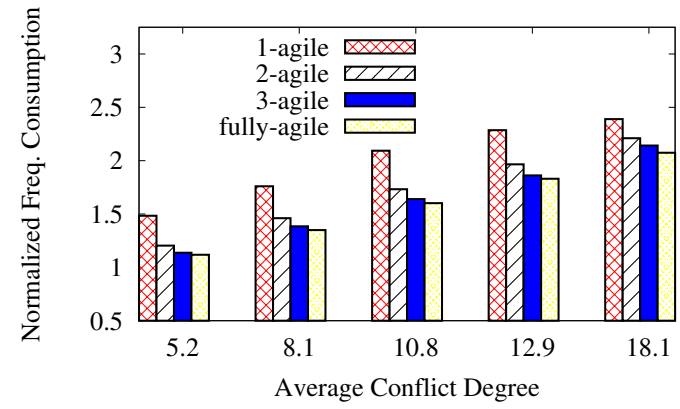

(b) Guard band size $=4$ segments per chunk

Fig. 9. The impact of frequency agility when both demand dynamics and conflict heterogeneity are present. Using 2-agile radios reduces the spectrum consumption by $12 \%-26 \%$ compared to 1 -agile radios. The improvement increases to $27 \%-40 \%$ when using fully-agile radios, but decreases in the presence of guard band overhead.

the rest in the $1000 m \times 1000 m$ area. We vary the proportion of nodes in the cluster and plot the result in Fig. 8(b). We observe that while the total frequency consumption increases with the density of the cluster, the benefit of radio agility remains stable, i.e. $10 \%-12 \%$.

These results, combined with our analytical results, show that using $k$-agile radios can lead to significant improvements under specific conflict topologies, but much smaller improvements under typical conflict topologies.

\section{FURTHER CONSIDERATION}

When Two Factors Combine. Having examined the impact of demand dynamics and conflict heterogeneity individually, we now examine the performance of agile radios when both factors are present.

We first present the analytical conclusions. Clearly the worst case results in previous sections continue to hold because the combined scenario is a generalization of the individual scenarios. In particular, Theorems 2 and 7 still hold. Namely, $k$-agile radios still reduce spectrum consumption by no more than a factor of $k$ compared to 1 -agile radios. The average case analysis in Sec. V-B, however, becomes very hard to extend due to the hardness in modeling conflict graph distributions.

For completeness we also use simulations to quantify the impact of frequency agility under the combined scenario. We combined the default settings of the previous simulations to configure the combined scenario. Fig. 9(a) plots the normalized spectrum consumption using 1 - and $k$-agile radios. We see that 2 -agile radios achieve an improvement of $12 \%-26 \%$ over 1-agile radios, and the improvement increases to $27 \%$ $40 \%$ using fully-agile radios. Even with a guard band size of 4 segments, Fig. 9(b) shows that the improvement for fullyagile radios becomes $15 \%-32 \%$.

An Alternative Performance Metric. Throughout this paper, we evaluate the spectrum usage performance using the metric $\Omega_{k}(\mathbb{S}, \mathbb{A}, \mathbb{G})$, the amount of spectrum required to support a given demand. While this metric allows a consistent comparison across different scenarios and makes the analytical problem tractable, it should not be interpreted as the only indication of practical system performance. For instance, in practical streaming applications where nodes share spectrum among a fixed set of segments, the disruption rate is a wellaccepted metric of user experience. The disruption rate refers to the proportion of time when a node is unable to acquire adequate spectrum to support its demand. High disruption rate implies lower application quality or additional complexity at higher protocol layers to mitigate its effect.

To examine the impact of radio agility using the disruption rate metric, we assume that nodes share a fixed number of 1000 spectrum segments and their traffic patterns follow the settings in Sec. V-C. Our experimental results show that when the normalized load is $0.75,2$-agile radios can reduce the disruption rate to $3 \%$, compared to the $11 \%$ of 1 -agile radios. Compared to the frequency consumption results, the application-performance improvement becomes much more visible. This observation also implies that the metrics considered in our paper are only a small sample set on the system performance. Depending on the applications, using radios with higher frequency-agility may generate more profound impact on the system and application performance.

\section{RELATED WORK}

Our work is inspired by and built on existing works on frequency-agile radios. A significant amount of work has been done towards dynamic spectrum access using 1-agile radios and contiguous spectrum access [4], [13], [14]. These works enjoy the advantage of easy implementation using commodity WiFi radios. Others examine the performance of dynamic spectrum allocation using fully-agile radios combined with both centralized and distributed algorithms [3], [24], [25], [26]. Also, spectrum auction schemes are designed for both 1-agile radios and fully-agile radios [27], [28]. Our main contribution in this paper is to understand the tradeoff between performance and complexity in the context of frequency-agility. We do so by evaluating the performance improvement of $k$-agile radios over 1 -agile radios for different $k$.

The advantage of frequency-agility has been explored in recent works. First, in the context of opportunistic spectrum access, recent work recognizes the advantage of fully-agile radios over 1-agile radios in terms of channel sensing overhead [16]. It shows that fully-agile radios will need to sense less number of channels because they are able to utilize small 
spectrum fragments discovered by spectrum sensing. Our work complements this effort by systematically studying the benefit in terms of the spectrum sharing efficiency. We also extend the investigation to general $k$ values. Second, prior work [2] has studied the problem of fragmentation with 1-agile radios and proposed to compensate it in the time-domain. The authors also examined the performance gap assuming unit-disk graph based conflict graphs. In comparison, our work analyzes the performance difference from a much broader perspective. We analytically quantify the impact for both general and special conflict topologies, for $k$-agile radios with general $k$, and from both the worst case and the average case point of view.

Finally, many recent works have designed prototypes and testbeds for frequency-agile radios [5], [7], [9], [29], [30], [31], [32]. These efforts recognize that increasing the frequency agility requires sophisticated hardware and software designs. Our study advances the state of the art by providing a quantitative understanding on the gain of increasing frequencyagility. It would be an interesting work to verify our results on these testbeds, particularly using 2 -agile radios.

\section{CONCLUSION}

To understand the need for additional frequency-agility, we develop a systematic framework to study the spectrum access performance of networks using $k$-agile radios rather than 1agile radios. We show that because of two inherent properties of dynamic spectrum sharing, $k$-agile radios can be highly advantageous. We analytically quantify the improvement under both properties, individually and when combined. Our results show that in the worst cases, fully-agile radios can lead to unbounded performance improvement, while in the average cases, the improvement becomes 10\%-40\% under typical traffic models and conflict conditions considered by our paper.

Perhaps the most important and interesting observation is that 2-agile radios can realize the majority of the improvement brought by fully-agile radios (unlimited $k$ ). This trend bounds not only the radio hardware complexity/cost, but also the extra spectrum overhead due to frequency guard bands placed at allocation boundaries. While our conclusions are derived analytically and via simulations, an interesting future work is to verify these results using large frequency-agile radio testbeds incorporating realistic traffic traces, interference measurements and primary users.

\section{ACKNOWLEDGEMENT}

This research was supported by NSF grants CNS-0832090 and CNS-0721961. We thank the anonymous reviewers and our Shepherd Prof. Petri Mähönen for their valuable suggestions.

\section{REFERENCES}

[1] I. F. Akyildiz, W. Y. Lee, M. Vuran, and S. Mohanty, "NeXt generation/dynamic spectrum access/cognitive radio wireless networks: A survey," Computer Networks Journal (Elsevier), Fall 2006.

[2] T. Moscibroda, R. Chandra, Y. Wu, S. Sengupta, P. Bahl, and Y. Yuan, "Load-aware spectrum distribution in wireless lans," in Proc. of ICNP, 2008.
[3] L. Yang, L. Cao, H. Zheng, and E. Belding, "Traffic-aware dynamic spectrum access," in Proc. of WICON, 2008.

[4] R. Chandra, R. Mahajan, T. Moscibroda, R. Raghavendra, and P. Bahl, "A case for adapting channel width in wireless networks," in Proc. of SIGCOMM, 2008

[5] J. Acharya, H. Viswanathan, and S. Venkatesan, "Timing acquisition for non-contiguous OFDM based dynamic spectrum access," in Proc. of DySPAN, 2008.

[6] K. Challapali, C. Cordeiro, and D. Birru, "Evolution of spectrum-agile cognitive radios: first wireless internet standard and beyond," in Proc. of WICON, 2006.

[7] P. D. Sutton, B. Ozgul, K. E. Nolan, and L. E. Doyle, "Bandwidthadaptive waveforms for dynamic spectrum access networks," in Proc. of DySPAN, 2008.

[8] A. M. Wyglinski, "Effects of bit allocation on non-contiguous multicarrier-based cogntiive radio transceivers," in Proc. of VTC, 2006.

[9] L. Yang, W. Hou, L. Cao, B. Y. Zhao, and H. Zheng, "Supporting demanding wireless applications with frequency-agile radios," in Proc. of NSDI, 2010.

[10] W. Hou, L. Yang, L. Zhang, X. Shan, and H. Zheng, "Understanding the impact of cross-band interference," in Proc. of ACM Coronet Workshop, 2009.

[11] H. Tang, "Some physical layer issues of wide-band cognitive radio system," in Proc. of IEEE DySPAN, November 2005.

[12] D. Burgkhardt, I. Cosovic, and F. Jondral, "Dynamic spectrum allocation by hierarchical resource trading," in IEEE VTC Spring, 2008, pp. 17961800.

[13] Y. Yuan, P. Bahl, R. Chandra, P. A. Chou, J. I. Ferrell, T. Moscibroda, S. Narlanka, and Y. Wu, "Knows: Kognitiv networking over white spaces," in Proc. of IEEE DySPAN, 2007.

[14] Y. Yuan, P. Bahl, R. Chandra, T. Moscibroda, S. Narlanka, and Y. Wu, "Allocating dynamic time-spectrum blocks in cognitive radio networks," in Proc. of MobiHoc, 2007.

[15] L. Yang, L. Cao, and H. Zheng, "Physical interference driven dynamic spectrum management," in Proc. of IEEE DySPAN, 2008.

[16] D. Xu, E. Jung, and X. Liu, "Optimal bandwidth selection in multichannel cognitive radio networks: How much is too much?" in Proc. of DySPAN, 2008.

[17] D. E. Knuth, The Art of Computer Programming, Vol. 1 (3rd ed.): Fundamental Algorithms. Addison-Wesley, 1973.

[18] P. R. Wilson, M. S. Johnstone, M. Neely, and D. Boles, "Dynamic storage allocation: A survey and critical review," in Proc. of the International Workshop on Memory Management, 1995.

[19] J. M. Robson, "An estimate of the store size necessary for dynamic storage allocation," Journal of the ACM, vol. 18, no. 3, pp. 416-423, 1971.

[20] S. Karpinski, E. M. Belding, and K. C. Almeroth, "Towards realistic models of wireless workload," in Proc. of the WiNMee Workshop, 2007.

[21] M. Larsen, J. Propp, and D. Ullman, "The fractional chromatic number of mycielski's graphs," J. Graph Theory, vol. 19, no. 3, pp. 411-416, 1995.

[22] J. Gergov, "Approximation algorithms for dynamic storage allocations," in Proc. of ESA, 1996.

[23] _ "Algorithms for compile-time memory optimization," in Proc. of SODA, 1999.

[24] L. Cao and H. Zheng, "Distributed rule-regulated spectrum sharing," IEEE Journal on Selected Areas in Communications, vol. 26, no. 1, pp. 130-145, 2007.

[25] W. Y. Lee and I. F.Akyildiz, "Joint spectrum and power allocation for inter-cell spectrum sharing in cognitive radio networks," in Proc. of DySPAN, 2008.

[26] H. Rahul, N. Kushman, D. Katabi, C. Sodini, and F. Edalat, "Learning to share: narrowband-friendly wideband networks," in Proc. of SIGCOMM, 2008.

[27] A. Subramanian, M. Al-Ayyoub, H. Gupta, S. Das, and M.M.Buddhikot, "Near-optimal dynamic spectrum allocation in cellular networks," in Proc. of DySPAN, 2008.

[28] Y. Wu, B. Wang, K. J. R. Liu, and T. C. Clancy, "A multi-winner cognitive spectrum auction framework with collusion-resistant mechanisms," in Proc. of DySPAN, 2008.

[29] X. Jing, S. Anaraman, M. A. Ergin, I. Seskara, and D. Raychaudhuri, "Distributed coordination schemes for multi-radio co-existence in dense spectrum environments: An experimental study on the ORBIT testbed," in Proc. of DySPAN, 2008. 
[30] G. J. Minden et al., "Kuar: A flexible software-defined radio development platform," in Proc. of DySPAN, 2007.

[31] K. Tan et al., "SORA: High performance software radio using general purpose multi-core processors," in Proc. of NSDI, 2009.

[32] "Wireless open-access research platform," http://warp.rice.edu/.

[33] E. R. Scheinerman and D. H. Ullman, Fractional Graph Theory: A Rational Approach to the Theory of Graphs. New York: WileyInterscience, 1997.

\section{APPENDIX}

\section{A. Proof of Theorem 4}

As shown in Fig. 10, we classify allocated chunks into 3 categories. Type $A$ chunks have their both neighbor chunks empty. Type $B$ chunks have one empty neighbor and one allocated neighbor. Type $C$ chunks have their both neighbor chunks allocated. We use $L_{A}, L_{B}, L_{C}$ to denote the number of $A, B$, and $C$ chunks respectively, and let $L=L_{A}+L_{B}+L_{C}$. We use $M$ to denote the number of empty chunks.

Assume the system reaches an equilibrium. We estimate the dynamics of the number of empty chunks $M$ caused by new system activities. The only two types of system activities are:

- Start a new request: The system finds $x_{k}$ chunks to satisfy the request. At least $x_{k}-1$ empty chunks disappear. Since an exact fit is very rare, we approximately consider that the $x_{k}$-th chunk is not an exact fit, thus will only shrink but not disappear. Therefore, we estimate the dynamics of $M$ by $M \leftarrow M-\left(x_{k}-1\right)$.

- End an existing request: The system reclaims $x_{k}$ chunks from an existing request. Assuming the types of the $x_{k}$ chunks are independent, we can estimate the dynamics of $M$ by $M \leftarrow M+x_{k} \cdot\left(L_{C} / L-L_{A} / L\right)$. This is because reclaiming a Type $A$ chunk will decrease $M$ by 1, reclaiming a Type $C$ chunk will increase $M$ by 1 , and reclaiming a Type $B$ chunk does not affect $M$.

When the system stabilizes, the requests start and end at equal rates. From the above dynamics analysis, we have $x_{k} \cdot\left(L_{C} / L-L_{A} / L\right)-\left(x_{k}-1\right)=0$, which is equal to

$$
L_{C}=\frac{x_{k}-1}{x_{k}} L+L_{A} .
$$

From (7) and $L=L_{A}+L_{B}+L_{C}$, we obtain

$$
\begin{gathered}
L=L_{A}+L_{B}+\left(\frac{x_{k}-1}{x_{k}} L+L_{A}\right), \text { thus } \\
\frac{1}{x_{k}} L=2 L_{A}+L_{B} .
\end{gathered}
$$

By analyzing the spectrum map (Fig. 10) and counting the number of empty chunks, we have (ignoring boundary conditions)

$$
M=\frac{1}{2}\left(2 L_{A}+L_{B}\right) .
$$

This is because each Type $A$ chunk is adjacent to 2 empty chunks and each Type $B$ chunk is adjacent to 1 empty chunk.

Now, compare (8) and (9) it is clear that $L=2 x_{k} \cdot M$. This means that the number of allocated chunks is $2 x_{k}$ times the number of unused chunks. Together with Assumption 2, this means that the total amount of unused fragments is $1 /\left(2 x_{k}\right)$ that of the allocated spectrum. Thus, the expected spectrum consumption $\mathbf{E}_{\mathbb{S}}\left[\Omega_{k}\left(\mathbb{S}, \mathbb{A}_{k}\right)\right]$ is approximately $\frac{2 x_{k}+1}{2 x_{k}} \mathbf{E}[\mathbb{S}]$.

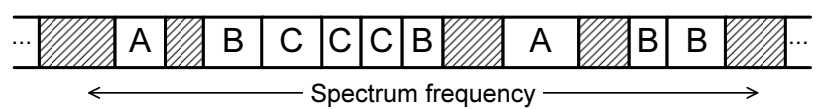

Fig. 10. An example spectrum allocation map. Allocated chunks are classified into three categories $(A, B$, and $C$ ). Empty chunks are shaded.

\section{B. Proof of Lemma 1}

We divide the proof into two steps:

Step 1: In this step, we prove that for any $\mathbb{G}$, there exists an integer $r \geq 1$, s.t. (5) is satisfied.

It has been shown that $\chi_{F}(\mathbb{G})=\min _{b} \frac{\chi_{b}(\mathbb{G})}{b}$ [33], where $\chi_{b}(\mathbb{G})$, the $b$-fold Chromatic Number, refers to the minimum number of colors needed to color $\mathbb{G}$ such that every node gets $b$ colors. In this case, the colors assigned to a node are not necessarily contiguous. Therefore, $\chi_{b}(\mathbb{G})=\Omega_{\infty}(\{b, b, \cdots, b\}, \mathbb{G})$. Let $r=\operatorname{argmin}_{b} \frac{\chi_{b}(\mathbb{G})}{b}$. We have $\chi_{F}(\mathbb{G})=\frac{\chi_{r}(\mathbb{G})}{r}$, and hence $\Omega_{\infty}(\{r, r, \cdots, r\}, \mathbb{G})=r \cdot \chi_{F}(\mathbb{G})$.

Step 2: We prove that for any $\mathbb{G}$ and any integer $r \geq 1$, (6) is always satisfied. Based on this result and the conclusion in Step 1, we can always find a $r$ that satisfies both (5) and (6), and complete the proof.

To prove (6), we first show $\Omega_{1}(\{r, r, \cdots, r\}, \mathbb{G}) \leq r \cdot \chi(\mathbb{G})$. Let $A(r, c)$ represent an allocation using 1 -agile radios that assigns $r$ segments to each node and uses $c$ segments in total. From the definition of $\chi(\mathbb{G})$ we know there exists an allocation $A(1, \chi(\mathbb{G}))$. By substituting each segment with $r$ new segments, we get an allocation $A^{\prime}(r, r \cdot \chi(\mathbb{G}))$.

Next, we show $\Omega_{1}(\{r, r, \cdots, r\}, \mathbb{G}) \geq r \cdot \chi(\mathbb{G})$. Assuming there is an allocation $A(r, c)$, we need to show that $c \geq$ $r \cdot \chi(\mathbb{G})$. To do so, we define an allocation to be aligned if every node's assigned segment set starts from $k r+1$ and ends at $(k+1) r$ for some integer $k \geq 0$. In other words, by binding every $r$ contiguous segments together $\{1,2, \cdots, r\}$, $\{r+1, r+2, \cdots, 2 r\}, \cdots$, each node will receive a set of $r$ contiguously-aligned segments. We now describe a procedure that can transform any allocation $A(r, c)$ to an aligned allocation $A^{\prime}\left(r, c^{\prime}\right)$ with $c^{\prime} \leq c$. Find the set of nodes $W$ who are assigned un-aligned segments. Find a node $n$ in $W$ whose assigned segments have the lowest index. Modify $n$ 's segment allocation so that $n$ takes the $r$ available segments with the lowest index. Because each of $n$ 's conflicting neighbors that occupy lower-indexed segments will have an aligned segment allocation, the above operation will provide $n$ an aligned segment allocation that is also conflict free. Repeat this process until all nodes have an aligned segment allocation.

It is clear that since the above transformation only moves node's segment allocation towards lower indices, it will result in an aligned allocation $A^{\prime}\left(r, c^{\prime}\right)$ with $c^{\prime} \leq c$. Now the aligned allocation $A^{\prime}\left(r, c^{\prime}\right)$ can be directly mapped into an allocation $A^{\prime \prime}\left(1,\left\lfloor\frac{c^{\prime}}{r}\right\rfloor\right)$, with segment $k$ assigned to a node in $A^{\prime \prime}$ iff segments $((k-1) r+1, k r)$ are assigned to it in $A^{\prime}$. Therefore, $\left\lfloor\frac{c^{\prime}}{r}\right\rfloor \geq \chi(\mathbb{G})$, and $c \geq c^{\prime} \geq r \cdot \chi(\mathbb{G})$. 